\title{
THE EXTENSION OF THE HUBBLE DIAGRAM. II. NEW REDSHIFTS AND PHOTOMETRY OF VERY DISTANT GALAXY CLUSTERS: FIRST INDICATION OF A DEVIATION OF THE HUBBLE DIAGRAM FROM A STRAIGHT LINE
}

\author{
Jerome Kristian, Allan Sandage, and James A. Westphal \\ Hale Observatories, Carnegie Institution of Washington, California Institute of Technology \\ Received 1977 July 22; accepted 1977 October 31
}

\begin{abstract}
Redshifts are given for 50 brightest cluster galaxies, extending as far as $z=0.75 ; B V R$ photometry is given for 33 clusters. These data are combined with earlier data of a similar kind in order to investigate several effects. The measured $B-V$ and $V-R$ colors as a function of redshift are well represented by Whitford's standard-galaxy $K$ corrections, as far as these are defined (to $z=0.28$ in $B-V$ and $z=0.48$ in $V-R$ ). This suggests both that the $K$ corrections are valid over these ranges of $z$ and that no major color change of the galaxies has occurred over the last 4-5 $\times 10^{9}$ years. At larger redshifts, the colors, which start out being monotonically redder with $z$, turn over and become bluer with $z$. The data at large $z$ seem to follow the prediction based upon ultraviolet photometry of NGC 4486 (M87), which is one extreme of a range of galaxies measured by Code and Welch.

Other standard corrections to the measurements are discussed, and formal least-squares values of $q_{0}$ are computed. To explore the possibility that one might be stalemated by the fact that the value of $q_{0}$ must, in principle, be known a priori to compute the aperture correction, the data are corrected separately for assumed $q_{0}$ values of 0 and +1 . The subsequently computed values of $q_{0}$ differ by only 0.2 between the two cases, which indicates that a simple iterative procedure will converge to produce a self-consistent value of $q_{0}$. The new data do not significantly change earlier discussions of the corrections for cluster richness and Bautz-Morgan contrast type. If these two corrections are not made, the effect on the present data is to significantly increase the positive curvature of the Hubble diagram, as well as the dispersion in apparent magnitude. To within the limits of the present data, the absolute magnitude of the brightest cluster galaxy does not, in general, depend on whether it is also a bright radio source.

The present sample, cut off at $z=0.4$ to avoid selection effects and uncertainties in the data, shows the first significant evidence for curvature of the Hubble diagram, with $V$ and $R$ magnitudes giving similar results. The formal value of $q_{0}$ (with galaxy evolution ignored) is $+1.6 \pm 0.4$. The dispersion in absolute magnitude is less than $0.3 \mathrm{mag}$, with $M_{V}=-23.28 \pm 0.03$ and $M_{R}=-24.09 \pm 0.03$.

The use of the Hubble diagram in cosmology now depends on a knowledge of brightness changes in galaxies, on the one hand, or of $q_{0}$ from other evidence, on the other. For example, if it were known with certainty that there has been no significant change in elliptical galaxy luminosities during the last $4 \times 10^{9}$ years, then the present data are nearly good enough for one to say definitively that the universe is closed and finite, with a finite lifetime. At the other extreme, if it were known with certainty from other evidence that the universe was nearly empty $\left(q_{0} \approx 0\right)$, then the present data set the constraint that net galaxy luminosities have decreased by $\sim 0.5$ mag during the last $5 \times 10^{9}$ years, with a net color change $\Delta(B-V) \leqslant 0.1$ mag. It seems possible at present to construct a self-consistent model with $q_{0} \approx 0$ that satisfies the known data, but the case is not yet settled.
\end{abstract}

Subject headings: cosmology — galaxies: clusters of — galaxies: redshifts

\section{INTRODUCTION}

This paper is the second in a series reporting measurements of redshifts and magnitudes of faint galaxies. The overall aim is to obtain these fundamental data for a large sample of galaxies from the former photographic cutoff at $z=0.2$ to as great a redshift as possible, in order to investigate problems relating to the evolution of galaxies and of the universe. We have used SIT television detectors for sky-subtraction spectroscopy and two-dimensional photometry, in addition to conventional photomultiplier aperture photometry. Our strategy has been to start at the bright end of the range $(z \approx 0.2)$ and to work toward fainter objects as experience is gained with the new detectors. Paper I (Sandage, Kristian, and Westphal 
TABLE 1

Redshifts Determined from 1975 June to 1976 June with the Digital Sky-Subtraction Spectrograph WITH SIT DETECTOR (Hale $5 \mathrm{~m}$ reflector)

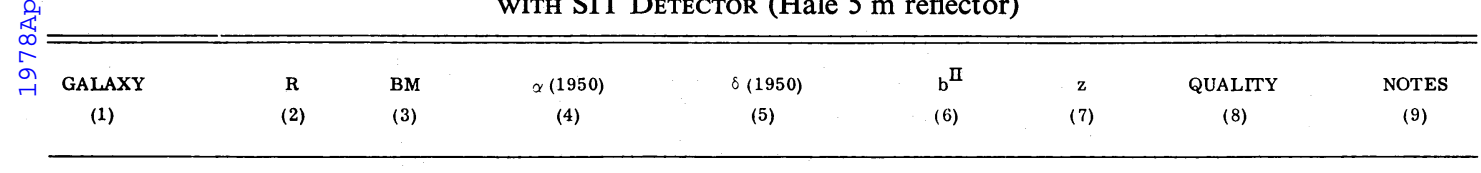

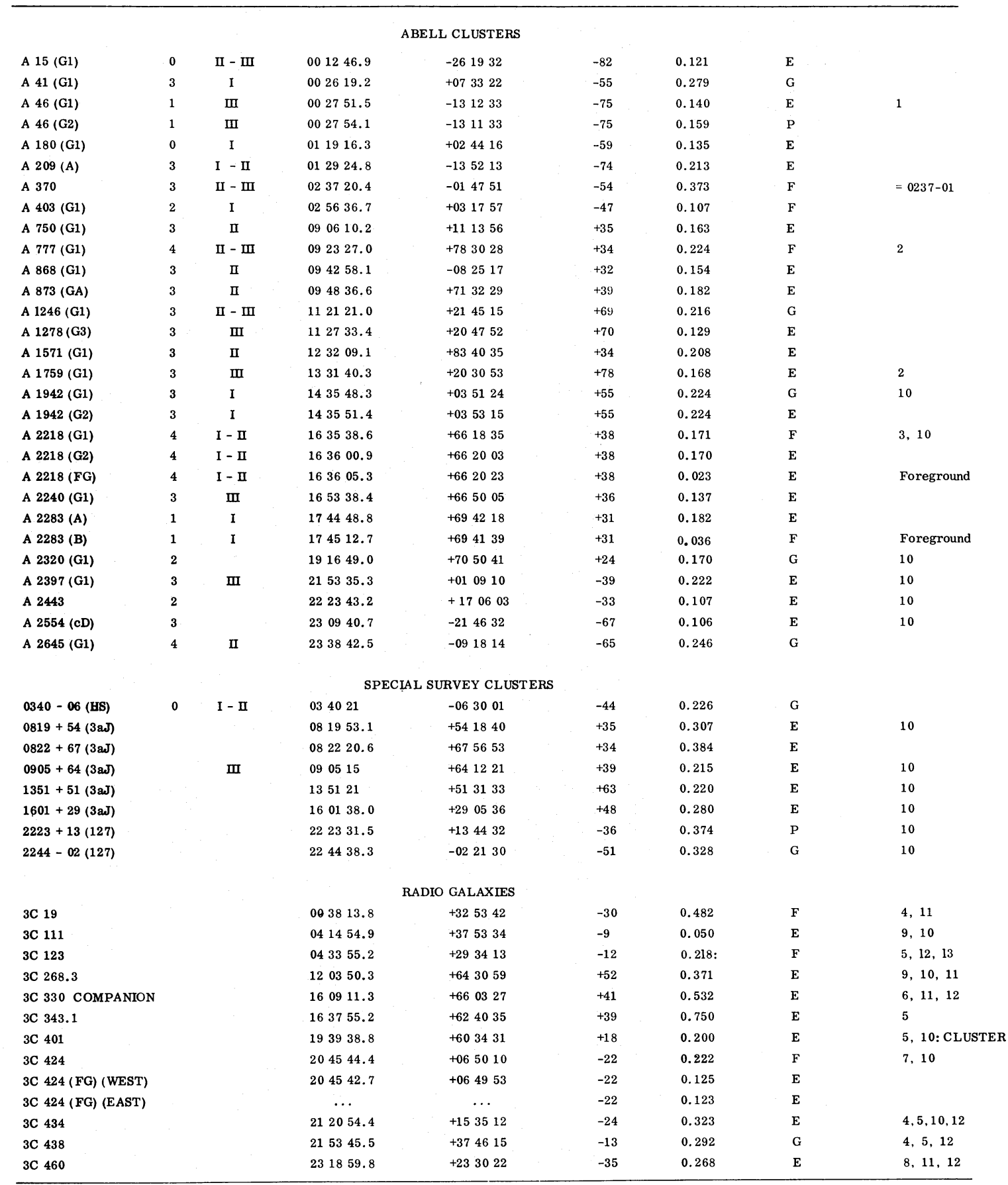




\section{NOTES TO TABLE 1}

1. A46. If G1 is not foreground, it is the brightest cluster member. It would be useful to have redshifts of other galaxies in the cluster, as well as confirmation of $\mathrm{G} 1$.

2. A777 and A1759. Redshifts of Paper I confirmed.

3. A2218. G1 is centrally located in the cluster. G2, which is more compact and probably brighter than $\mathrm{G} 1$, is near the bright foreground spiral. The latter, which appears somewhat disturbed on direct plates, has bright Balmer-line emission.

4. Possible cluster.

5. Independent redshift by Spinrad and Smith. 3C 343.1 has been discussed in some detail by Spinrad et al. 1977 .

6. 3C 330. The redshift is for the northern galaxy of the pair near the center of the radio source (Kristian, Sandage, and Katem 1978), on the basis of $\lambda 3727$ emission and $H$ and $\mathrm{K}$ absorption. The southern galaxy of the pair, which is almost certainly the radio source, has a rich emission-line spectrum with $z=0.549$ (Spinrad et al. 1976). The difference may be marginally real, but it is small enough that there is no doubt that these two galaxies are the brightest in a cluster.

7. 3C 424. The identification of the radio source is the galaxy shown by Wyndham 1966, for which we measure $z=0.222$. There are a number of other galaxies in the vicinity, some of them brighter than the radio galaxy, which could be a cluster. The brightest of these is a close pair (FG1 and FG2:

1976) summarized the observations from 1974 October to 1975 June and discussed the cluster richness correction. The present paper gives the observational results from 1975 June to 1976 June. The new data fill in the Hubble diagram significantly in the redshift range $z=0.25-0.5$ and extend as far as $z=0.75$. Since the sample is not likely to be extended very rapidly at the faint end, we have taken the occasion to perform an interim formal solution for $q_{0}$, ignoring possible galaxy evolution effects. The result of this analysis is the first indication in our data of a deviation of the Hubble diagram from a straight line, with a formal value for $q_{0}$ of about +1.6 .

\section{NEW REDSHIFTS AND PHOTOMETRY}

Table 1 gives new redshifts for 50 galaxies measured with the SIT-prism spectrograph at the Cassegrain focus of the $5 \mathrm{~m}$ Hale telescope (Westphal, Kristian, and Sandage 1975). Almost all of the objects measured are the brightest galaxies in clusters: either Abell clusters, clusters found on a special 48 inch $(1.2 \mathrm{~m})$ Schmidt survey using IIIa-J or 127-04 emulsions, or clusters containing $3 \mathrm{C}$ radio sources.

Table 1 includes the Abell richness class (R) and Bautz-Morgan contrast class (BM) of the clusters, and the quality of the spectra (col. [8]: $\mathrm{E}=$ excellent, $\mathrm{G}=$ good, $\mathrm{F}=$ fair, and $\mathrm{P}=$ poor). The positions listed in columns (4) and (5) are of the objects whose redshifts are given. The positions should be accurate to better than $15^{\prime \prime}$, which is good enough to provide a unique identification.

Twenty-eight of the redshifts are greater than 0.2 , of which seven are in the range 0.3-0.4 and three are greater than 0.4. The largest redshifts are all for $3 \mathrm{C}$ sources (3C 19, $z=0.48$; 3C 330, $z=0.53$; and $3 \mathrm{C} 343.1, z=0.75$ ). This is due to a very strong selection effect: Extremely faint $3 \mathrm{C}$ sources have been searched for intensively by several groups for some years.
Separation is $\sim 5^{\prime \prime}$; central position is given in Table 1) with $z=0.124$. Whether FG1 and FG2 are the bright members of a cluster with 3C 424 a background object, or 3C 424 is in the cluster with FG1 and FG2 foreground objects, or there is not a cluster but a chance clumping of field galaxies will not be known until redshifts of more of the galaxies are measured.

8. 3C 460. Possible cluster. Earlier scanner redshift (Kristian, Sandage, and Katem 1974) confirmed.

9. 3C 111 and 3C 268.3. Very bright emission-line spectra: $\lambda 3727, \mathrm{H} \beta, \lambda 4959, \lambda 5007, \mathrm{H} \gamma .3 \mathrm{C} 111$ has extremely strong $\mathbf{H} \alpha$, strong red continuum; independent redshift by J. B. Oke and W. L. W. Sargent (quoted in Longair and Gunn 1975). $3 \mathrm{C} 268.3$ is in a distant cluster.

10. No photometry available.

11. See Kristian, Sandage, and Katem 1974.

12. See Kristian, Sandage, and Katem 1978.

13. 3C 123. A redshift of 0.637 was originally suggested by Spinrad 1975, primarily on the basis of an emission line at observed wavelength $\lambda 6100$, interpreted as rest wavelength 13727. A lower-quality SIT-prism spectrogram shows the same line and the same general features. More recent scans in the infrared by Spinrad (private communication) indicate that this line is $\lambda 5007$ and that the redshift of $3 \mathrm{C} 123$ is 0.218 .

We have new photometry for 33 clusters. Table 2 lists conventional photomultiplier results for 26 clusters (as well as one quasar), obtained with the $5 \mathrm{~m}$ telescope. The redshifts given in column (3) are from Table 1, unless otherwise noted. In most cases, measurements were made through two or three apertures (col. [5]) to estimate the validity of the aperture correction. The magnitudes and colors listed in columns (6)-(10) are those measured through the corresponding apertures, with no corrections of any kind other than for atmospheric extinction.

Table 3 gives photometry for seven clusters, obtained with a two-dimensional SIT detector at the prime focus of the $5 \mathrm{~m}$ telescope (Westphal and Kristian 1976). The magnitudes given are those within the listed diameters. The notation " $\operatorname{SM}(x)$ " means that the corresponding diameter ( $x$ arcsec) is the standard metric diameter for the appropriate redshift, as defined by Sandage (1972a). The SIT measurements have been reduced to the photoelectric $B V R$ system of Table 2. Redshifts are from Table 1, if not stated otherwise in the notes to Table 3 . The accuracy of the SIT photometry is of order $0.05 \mathrm{mag}$ for each passband.

\section{THE COLOR-REDSHIFT RELATIONS}

Figures 1 and 2 show the relation between observed $B-V$ and $V-R$ colors (corrected for galactic reddening [Sandage 1973a]) and redshifts for the new data of Tables 2 and 3 combined with older data of Paper I and of Sandage (1973a, first part of his Table 6). The solid lines in Figure 2, and in Figure 1 to the left of the fork at $z=0.28$, are from Whitford's (1975) $K$ corrections for a standard elliptical galaxy.

The two lines to the right of $z=0.28$ in Figure 1 are from Code and Welch (1977), based on ultraviolet photometry from the Orbiting Astronomical Observatory. Code and Welch find that there is a scatter of ultraviolet intensity distributions in early-type galaxies 
which becomes progressively larger at shorter wavelengths. The two curves in Figure 1 represent the extremes for the Code and Welch data: the center of I. M31 (top curve) and NGC 4486 (bottom curve). The former flattens out at $z=0.25$ and then begins to turn redward again past $z=0.5$, while the latter turns over at $z=0.25$ and becomes increasingly bluer with larger redshift. Our $B-V$ color data are not very accurate or very numerous beyond $z=0.3$, and are certainly not definitive, but they appear to be incompatible with the M31 curve and not an unreasonable fit to the NGC 4486 curve. The same may perhaps be said of the $V-R$ data in Figure 2, which show a similar turnover and increasing blueness with redshifts past $z=0.5$, although the data are even fewer and weaker. A turnover of colors at large redshift

TABLE 2

S-20 Photoelectric Photometry

\begin{tabular}{|c|c|c|c|c|c|c|c|c|c|}
\hline $\begin{array}{c}\text { CLUSTER } \\
\text { (1) }\end{array}$ & $\begin{array}{c}\text { OBJECT } \\
\text { (2) }\end{array}$ & (3) & $b^{I I}$ & $\begin{array}{l}\text { Apert. } \\
\text { (arc } \\
\text { sec) } \\
(5)\end{array}$ & $\begin{array}{c}\mathrm{B} \\
\mathrm{mag} \\
(6)\end{array}$ & $\begin{array}{c}\mathrm{V} \\
\mathrm{mag} \\
(7)\end{array}$ & $\begin{array}{c}\mathrm{R} \\
\mathrm{mag} \\
(8)\end{array}$ & $\begin{array}{c}\mathrm{B}-\mathrm{V} \\
\mathrm{mag} \\
(9)\end{array}$ & $\begin{array}{l}\mathrm{V}-\mathrm{R} \\
\mathrm{mag} \\
(10)\end{array}$ \\
\hline \multirow[t]{2}{*}{ A 15} & G1 & 0.121 & -82 & $\begin{array}{r}7.6 \\
12.2\end{array}$ & $\begin{array}{l}18.62 \pm .014 \\
18.29 \pm .026\end{array}$ & $\begin{array}{l}17.11 \pm .014 \\
16.81 \pm .013\end{array}$ & $\begin{array}{l}16.19 \pm .011 \\
15.85 \pm .018\end{array}$ & $\begin{array}{l}1.51 \pm .017 \\
1.48 \pm .029\end{array}$ & $\begin{array}{l}0.92 \pm .014 \\
0.96 \pm .022\end{array}$ \\
\hline & G2 & & & 12.2 & $18.50 \pm .020$ & $17.30 \pm .014$ & $16.46 \pm .021$ & $1.20 \pm .024$ & $0.84 \pm .025$ \\
\hline A 41 & A & 0.279 & -55 & $\begin{array}{l}12.2 \\
18.8\end{array}$ & $\begin{array}{c}19.87 \pm .043 \\
\ldots\end{array}$ & $\begin{array}{l}18.24 \pm .022 \\
18.03 \pm .062\end{array}$ & $\begin{array}{l}17.02 \pm .014 \\
16.78 \pm .025\end{array}$ & $\begin{array}{c}1.63 \pm .048 \\
\ldots\end{array}$ & $\begin{array}{l}1.22 \pm .026 \\
1.25 \pm .067\end{array}$ \\
\hline \multirow[t]{3}{*}{ A 46} & G1 & 0.140 & -75 & $\begin{array}{l}12.2 \\
18.8\end{array}$ & $\begin{array}{l}18.81 \pm .024 \\
18.37 \pm .041\end{array}$ & $\begin{array}{l}17.28 \pm .017 \\
17.04 \pm .019\end{array}$ & $\begin{array}{l}16.35 \pm .012 \\
16.05 \pm .040\end{array}$ & $\begin{array}{l}1.53 \pm .029 \\
1.33 \pm .045\end{array}$ & $\begin{array}{l}0.93 \pm .021 \\
0.99 \pm .044\end{array}$ \\
\hline & G2 & $0.159:$ & & $\begin{array}{l}12.2 \\
18.8\end{array}$ & $\begin{array}{c}19.67 \pm .040 \\
\ldots\end{array}$ & $\begin{array}{l}18.02 \pm .026 \\
17.67 \pm .038\end{array}$ & $\begin{array}{l}17.05 \pm .025 \\
16.77 \pm .035\end{array}$ & $\begin{array}{c}1.65 \pm .048 \\
\ldots\end{array}$ & $\begin{array}{l}0.97 \pm .036 \\
0.90 \pm .052\end{array}$ \\
\hline & G3 & & & 12.2 & $\cdots$ & $17.96 \pm .023$ & $17.02 \pm .022$ & $\ldots$ & $0.94 \pm .032$ \\
\hline A 180 & G1 & 0.135 & -59 & $\begin{array}{l}18.8 \\
30.6\end{array}$ & $\begin{array}{c}18.07 \pm .020 \\
\ldots\end{array}$ & $\begin{array}{l}16.66 \pm .012 \\
16.43 \pm .021\end{array}$ & $\begin{array}{l}15.66 \pm .020 \\
15.48 \pm .022\end{array}$ & $\begin{array}{c}1.41 \pm .023 \\
\ldots\end{array}$ & $\begin{array}{l}1.00 \pm .023 \\
0.95 \pm .030\end{array}$ \\
\hline A 209 & A & 0.213 & -74 & $\begin{array}{l}12.2 \\
18.8\end{array}$ & $18 . \ddot{60} \pm .070$ & $\begin{array}{l}17.29 \pm .017 \\
16.91 \pm .019\end{array}$ & $\begin{array}{l}16.29 \pm .019 \\
15.90 \pm .018\end{array}$ & $1.69 \pm .073$ & $\begin{array}{l}1.00 \pm .025 \\
1.01 \pm .026\end{array}$ \\
\hline \multirow[t]{2}{*}{ A 370} & N8 & 0.373 & -54 & $\begin{array}{l}12.2 \\
12.2 \\
18.8\end{array}$ & $\begin{array}{l}20.35 \pm .078 \\
20.56 \pm .072 \\
\quad \cdots\end{array}$ & $\begin{array}{l}18.85 \pm .050 \\
18.96 \pm .037 \\
18.64 \pm .067\end{array}$ & $\begin{array}{l}17.42 \pm .047 \\
17.58 \pm .031 \\
17.28 \pm .035\end{array}$ & $\begin{array}{l}1.50 \pm .093 \\
1.60 \pm .081 \\
\ldots\end{array}$ & $\begin{array}{l}1.43 \pm .069 \\
1.38 \pm .048 \\
1.39 \pm .076\end{array}$ \\
\hline & N9 & & & $\begin{array}{l}12.2 \\
18.8\end{array}$ & $19.96 \pm .076$ & $\begin{array}{l}18.87 \pm .052 \\
18.46 \pm .050\end{array}$ & $\begin{array}{l}17.46 \pm .033 \\
16.97 \pm .039\end{array}$ & $1 . \ddot{50 \pm .091}$ & $\begin{array}{l}1.41 \pm .062 \\
1.49 \pm .063\end{array}$ \\
\hline A 403 & G1 & 0.107 & -47 & $\begin{array}{l}12.2 \\
18.8\end{array}$ & $\begin{array}{l}18.17 \pm .015 \\
17.67 \pm .018\end{array}$ & $\begin{array}{l}16.78 \pm .011 \\
16.31 \pm .016\end{array}$ & $\begin{array}{l}15.80 \pm .013 \\
15.32 \pm .011\end{array}$ & $\begin{array}{l}1.39 \pm .019 \\
1.36 \pm .024\end{array}$ & $\begin{array}{l}0.99 \pm .017 \\
0.98 \pm .019\end{array}$ \\
\hline \multirow[t]{3}{*}{ A 655} & G1 & $\begin{array}{l}0.124 \\
\text { (Paper I) }\end{array}$ & +35 & $\begin{array}{l}12.2 \\
18.8 \\
30.6\end{array}$ & $\begin{array}{l}17.41 \pm .009 \\
17.07 \pm .014 \\
16.84 \pm .015\end{array}$ & $\begin{array}{l}15.93 \pm .008 \\
15.62 \pm .008 \\
15.35 \pm .009\end{array}$ & $\begin{array}{l}14.96 \pm .005 \\
14.63 \pm .007 \\
14.38 \pm .007\end{array}$ & $\begin{array}{l}1.48 \pm .012 \\
1.45 \pm .016 \\
1.49 \pm .017\end{array}$ & $\begin{array}{l}0.97 \pm .009 \\
0.99 \pm .011 \\
0.97 \pm .011\end{array}$ \\
\hline & G2 & & & $\begin{array}{l}12.2 \\
18.8 \\
30.6\end{array}$ & $\begin{array}{l}15.22 \pm .021 \\
18.19 \pm .023 \\
18.00 \pm .029\end{array}$ & $\begin{array}{l}16.83 \pm .012 \\
16.61 \pm .022 \\
16.42 \pm .021\end{array}$ & $\begin{array}{l}15.82 \pm .009 \\
15.57 \pm .012 \\
15.36 \pm .016\end{array}$ & $\begin{array}{l}1.61 \pm .024 \\
1: 58 \pm .032 \\
1.58 \pm .036\end{array}$ & $\begin{array}{l}1.01 \pm .015 \\
1.04 \pm .025 \\
1.06 \pm .026\end{array}$ \\
\hline & G3 & & & $\begin{array}{l}12.2 \\
18.8\end{array}$ & $\begin{array}{l}18.57 \pm .022 \\
18.34 \pm .036\end{array}$ & $\begin{array}{l}17.17 \pm .015 \\
16.95 \pm .021\end{array}$ & $\begin{array}{l}16.23 \pm .014 \\
16.05 \pm .015\end{array}$ & $\begin{array}{l}1.40 \pm .027 \\
1.39 \pm .042\end{array}$ & $\begin{array}{l}0.94 \pm .021 \\
0.90 \pm .026\end{array}$ \\
\hline A 665 & G1 & $\begin{array}{l}0.183 \\
\text { (Sargent) }\end{array}$ & +35 & $\begin{array}{l}12.2 \\
18.8\end{array}$ & $\begin{array}{l}19.08 \pm .027 \\
18.62 \pm .026\end{array}$ & $\begin{array}{l}17.48 \pm .022 \\
17.04 \pm .029\end{array}$ & $\begin{array}{l}16.43 \pm .022 \\
16.02 \pm .025\end{array}$ & $\begin{array}{l}1.60 \pm .035 \\
1.58 \pm .039\end{array}$ & $\begin{array}{l}1.05 \pm .031 \\
1.02 \pm .038\end{array}$ \\
\hline A 750 & G1 & 0.163 & +35 & $\begin{array}{l}12.2 \\
18.8 \\
30.6\end{array}$ & $\begin{array}{l}18.63 \pm .024 \\
18.17 \pm .026 \\
17.75 \pm .028\end{array}$ & $\begin{array}{l}17.03 \pm .020 \\
16.63 \pm .018 \\
16.27 \pm .026\end{array}$ & $\begin{array}{l}16.01 \pm .017 \\
15.54 \pm .014 \\
15.26 \pm .016\end{array}$ & $\begin{array}{l}1.60 \pm .031 \\
1.54 \pm .032 \\
1.48 \pm .038\end{array}$ & $\begin{array}{l}1.02 \pm .026 \\
1.09 \pm .023 \\
1.01 \pm .031\end{array}$ \\
\hline A 868 & G1 & 0.154 & +32 & $\begin{array}{l}12.2 \\
18.8\end{array}$ & $\begin{array}{l}18.69 \pm .024 \\
18.26 \pm .024\end{array}$ & $\begin{array}{l}17.23 \pm .021 \\
16.86 \pm .026\end{array}$ & $\begin{array}{l}16.18 \pm .016 \\
15.83 \pm .016\end{array}$ & $\begin{array}{l}1.46 \pm .032 \\
1.40 \pm .035\end{array}$ & $\begin{array}{l}1.05 \pm .026 \\
1.03 \pm .031\end{array}$ \\
\hline A 873 & A & 0.182 & +39 & $\begin{array}{l}12.2 \\
18.8\end{array}$ & $\begin{array}{l}19.13 \pm .044 \\
18.83 \pm .035\end{array}$ & $\begin{array}{l}17.62 \pm .023 \\
17.40 \pm .042\end{array}$ & $\begin{array}{l}16.55 \pm .023 \\
16.33 \pm .019\end{array}$ & $\begin{array}{l}1.51 \pm .050 \\
1.43 \pm .055\end{array}$ & $\begin{array}{l}1.07 \pm .033 \\
1.07 \pm .046\end{array}$ \\
\hline \multirow[t]{4}{*}{$\begin{array}{l}\text { A } 1093 \\
=1044+0918\end{array}$} & C & & & $\begin{array}{l}12.2 \\
18.8\end{array}$ & $\begin{array}{c}20.05 \pm .067 \\
\ldots\end{array}$ & $\begin{array}{l}18.71 \pm .054 \\
18.77 \pm .085\end{array}$ & $\begin{array}{l}17.81 \pm .044 \\
17.66 \pm .079\end{array}$ & $\begin{array}{l}1.34 \pm .086 \\
\ldots\end{array}$ & $\begin{array}{l}0.90 \pm .070 \\
1.11 \pm .116\end{array}$ \\
\hline & B & & & 18.8 & $19.11 \pm .072$ & $18.59 \pm .053$ & $17.86 \pm .100$ & $0.52 \pm .072$ & $0.73 \pm .113$ \\
\hline & $\mathbf{E}$ & & & 18.8 & $\cdots$ & $\cdots$ & $17.19 \pm .050$ & $\cdots$ & $\cdots$ \\
\hline & $\mathbf{x}$ & $\begin{array}{c}0.226 \\
\text { (Paper I) }\end{array}$ & +60 & 18.8 & $19.68 \pm .091$ & $17.96 \pm .058$ & $16.98 \pm .050$ & $1.72 \pm .108$ & $0.98 \pm .077$ \\
\hline A 1246 & G1 & 0.216 & +69 & $\begin{array}{l}12.2 \\
18.8\end{array}$ & $\begin{array}{l}18.76 \pm .029 \\
18.45 \pm .025\end{array}$ & $\begin{array}{l}17.16 \pm .017 \\
16.89 \pm .029\end{array}$ & $\begin{array}{l}16.10 \pm .019 \\
15.85 \pm .013\end{array}$ & $\begin{array}{l}1.60 \pm .034 \\
1.56 \pm .038\end{array}$ & $\begin{array}{l}1.06 \pm .025 \\
1.04 \pm .032\end{array}$ \\
\hline A 1278 & G1 & $\begin{array}{l}0.129 \\
\text { (G3) }\end{array}$ & +70 & $\begin{array}{l}12.2 \\
18.8 \\
30.6\end{array}$ & $\begin{array}{l}18.34 \pm .023 \\
18.05 \pm .020 \\
17.86 \pm .035\end{array}$ & $\begin{array}{l}16.92 \pm .013 \\
16.64 \pm .016 \\
16.45 \pm .015\end{array}$ & $\begin{array}{l}15.97 \pm .010 \\
15.72 \pm .023 \\
15.52 \pm .018\end{array}$ & $\begin{array}{l}1.42 \pm .026 \\
1.41 \pm .026 \\
1.41 \pm .038\end{array}$ & $\begin{array}{l}0.95 \pm .016 \\
0.92 \pm .028 \\
0.93 \pm .023\end{array}$ \\
\hline A 1571 & G1 & 0.208 & +34 & $\begin{array}{l}18.8 \\
30.6 \\
\end{array}$ & $\begin{array}{l}19.39 \pm .059 \\
\ldots \\
\end{array}$ & $\begin{array}{l}17.72 \pm .036 \\
17.43 \pm .063 \\
\end{array}$ & $\begin{array}{l}16.44 \pm .026 \\
16.20 \pm .034 \\
\end{array}$ & $\begin{array}{l}1.67 \pm .069 \\
\quad \ldots \\
\end{array}$ & $\begin{array}{l}1.28 \pm .044 \\
1.23 \pm .072 \\
\end{array}$ \\
\hline
\end{tabular}


TABLE 2-Continued

\begin{tabular}{|c|c|c|c|c|c|c|c|c|c|}
\hline $\begin{array}{c}\text { CLUSTER } \\
\text { (1) }\end{array}$ & OBJECT & (3) & $\begin{array}{l}b^{\text {II }} \\
\text { (4) }\end{array}$ & $\begin{array}{l}\text { Apert. } \\
\text { (arc } \\
\text { sec) } \\
(5)\end{array}$ & $\begin{array}{c}\mathrm{B} \\
\mathrm{mag} \\
(6)\end{array}$ & $\begin{array}{c}\mathrm{V} \\
\mathrm{mag} \\
(7)\end{array}$ & $\begin{array}{c}\mathrm{R} \\
\mathrm{mag} \\
(8)\end{array}$ & $\begin{array}{l}\mathrm{B}-\mathrm{V} \\
\mathrm{mag} \\
(9)\end{array}$ & $\begin{array}{l}\mathrm{V}-\mathrm{R} \\
\mathrm{mag} \\
(10)\end{array}$ \\
\hline $\begin{array}{l}\text { A } 1878 \\
=1411+2928\end{array}$ & $\mathrm{~A} 1$ & $\begin{array}{c}0.222 \\
\text { (Paper I) }\end{array}$ & +72 & $\begin{array}{l}18.8 \\
30.6\end{array}$ & $\begin{array}{c}19.29 \pm .040 \\
\ldots\end{array}$ & $\begin{array}{l}17.63 \pm .030 \\
17.33 \pm .045\end{array}$ & $\begin{array}{l}16.48 \pm .017 \\
16.28 \pm .092\end{array}$ & $\begin{array}{l}1.66 \pm .050 \\
\ldots\end{array}$ & $\begin{array}{l}1.15 \pm .034 \\
1.05 \pm .102\end{array}$ \\
\hline $\begin{array}{l}\text { A } 1952 \\
=1438+2850\end{array}$ & $\mathrm{~B}$ & $\begin{array}{l}0.247 \\
\text { (Paper I) }\end{array}$ & +66 & $\begin{array}{l}12.2 \\
18.8\end{array}$ & $\begin{array}{l}20.19 \pm .057 \\
19.63 \pm .075\end{array}$ & $\begin{array}{l}18.58 \pm .039 \\
17.94 \pm .051\end{array}$ & $\begin{array}{l}17.41 \pm .030 \\
16.88 \pm .026\end{array}$ & $\begin{array}{l}1.61 \pm .069 \\
1.69 \pm .091\end{array}$ & $\begin{array}{l}1.17 \pm .049 \\
1.06 \pm .057\end{array}$ \\
\hline \multirow[t]{2}{*}{ A 2240} & G1 & 0.137 & +36 & $\begin{array}{l}12.2 \\
18.8\end{array}$ & $\begin{array}{l}18.56 \pm .055 \\
18.39 \pm .013\end{array}$ & $\begin{array}{l}17.12 \pm .019 \\
17.02 \pm .044\end{array}$ & $\begin{array}{l}16.24 \pm .029 \\
16.07 \pm .020\end{array}$ & $\begin{array}{l}1.44 \pm .058 \\
1.37 \pm .046\end{array}$ & $\begin{array}{l}0.88 \pm .035 \\
0.95 \pm .048\end{array}$ \\
\hline & G2 & & & 12.2 & $18.81 \pm .073$ & $17.35 \pm .027$ & $16.36 \pm .015$ & $1.46 \pm .078$ & $0.99 \pm .031$ \\
\hline \multirow[t]{2}{*}{ A 2283} & A & 0.182 & +31 & $\begin{array}{l}12.2 \\
18.8\end{array}$ & $\begin{array}{l}19.03 \pm .059 \\
18.50 \pm .062\end{array}$ & $\begin{array}{l}17.32 \pm .026 \\
16.95 \pm .031\end{array}$ & $\begin{array}{l}16.30 \pm .017 \\
15.90 \pm .017\end{array}$ & $\begin{array}{l}1.71 \pm .064 \\
1.55 \pm .069\end{array}$ & $\begin{array}{l}1.02 \pm .031 \\
1.05 \pm .035\end{array}$ \\
\hline & $\begin{array}{l}\text { B } \\
\text { Forgd. }\end{array}$ & 0.036 & & $\begin{array}{l}12.2 \\
18.8\end{array}$ & $\begin{array}{c}17.85 \pm .017 \\
\ldots\end{array}$ & $\begin{array}{c}16.78 \pm .020 \\
\ldots\end{array}$ & $\begin{array}{l}15.91 \pm .015 \\
15.63 \pm .020\end{array}$ & $\begin{array}{l}1.07 \pm .026 \\
\ldots\end{array}$ & $\begin{array}{c}0.87 \pm .025 \\
\ldots\end{array}$ \\
\hline A 2645 & G1 & 0.246 & -65 & $\begin{array}{l}12.2 \\
18.8\end{array}$ & $\begin{array}{l}19.96 \pm .046 \\
19.57 \pm .046\end{array}$ & $\begin{array}{l}18.24 \pm .024 \\
17.81 \pm .024\end{array}$ & $\begin{array}{l}17.01 \pm .020 \\
16.62 \pm .017\end{array}$ & $\begin{array}{l}1.72 \pm .052 \\
1.76 \pm .052\end{array}$ & $\begin{array}{l}1.23 \pm .039 \\
1.19 \pm .029\end{array}$ \\
\hline $0112+06$ & G3 & no $z$ & -57 & $\begin{array}{l}12.2 \\
18.8\end{array}$ & $\begin{array}{c}19.59 \pm .040 \\
\ldots\end{array}$ & $\begin{array}{l}17.95 \pm .021 \\
17.71 \pm .023\end{array}$ & $\begin{array}{l}16.95 \pm .018 \\
16.66 \pm .028\end{array}$ & $\begin{array}{c}1.64 \pm .045 \\
\ldots\end{array}$ & $\begin{array}{l}1.00 \pm .028 \\
1.05 \pm .036\end{array}$ \\
\hline \multirow[t]{2}{*}{ 0340-06 } & $\begin{array}{l}\text { A } \\
\text { Prob. } c\end{array}$ & $\begin{array}{l}0.226 \\
\mathrm{~m} .\end{array}$ & -44 & $\begin{array}{r}7.6 \\
18.8\end{array}$ & $\begin{array}{l}20.21 \pm .054 \\
18.94 \pm .071\end{array}$ & $\begin{array}{l}18.57 \pm .024 \\
17.33 \pm .025\end{array}$ & $\begin{array}{l}17.47 \pm .016 \\
16.29 \pm .020\end{array}$ & $\begin{array}{l}1.64 \pm .059 \\
1.61 \pm .075\end{array}$ & $\begin{array}{l}1.10 \pm .029 \\
1.04 \pm .032\end{array}$ \\
\hline & B & 0.226 & -44 & 12.2 & $\cdots$ & $19.04 \pm .042$ & $17.96 \pm .038$ & $\cdots$ & $1.08 \pm .057$ \\
\hline $3 C 19$ & & 0.482 & -30 & $\begin{array}{l}12.2 \\
12.2 \\
18.8\end{array}$ & $\begin{array}{l}\cdots \\
\cdots \\
\cdots\end{array}$ & $\begin{array}{l}20.53 \pm .10 \\
20.40 \pm .07 \\
20.55 \pm .15\end{array}$ & $\begin{array}{l}18.87 \pm .05 \\
\ldots . . \pm .10\end{array}$ & $\begin{array}{l}\cdots \\
\cdots \\
\cdots\end{array}$ & $\begin{array}{c}1.66 \pm .11 \\
\ldots . .18\end{array}$ \\
\hline 3C 123 & & $0.218:$ & -12 & $\begin{array}{l}12.2 \\
18.8\end{array}$ & $\cdots$ & $19 . \ddot{85} \pm .18$ & $\begin{array}{l}18.79 \pm .05 \\
18.27 \pm .08\end{array}$ & $\cdots$ & $\stackrel{\ldots}{1.58 \pm .20}$ \\
\hline $\begin{array}{l}\text { 3C 343* } \\
\text { (\$SO) }\end{array}$ & & 0.988 & +39 & 7.6 & $21.06 \pm .085$ & $20.61 \pm .09$ & $19.55 \pm .035$ & $0.45 \pm .13$ & $1.06 \pm .10$ \\
\hline $3 \mathrm{C} 343.1$ & & 0.75 & +39 & 7.6 & $21.90 \pm .09$ & $20.74 \pm .09$ & $19.65 \pm .05$ & $1.16 \pm .13$ & $1.09 \pm .10$ \\
\hline
\end{tabular}

* Note on 3C 343. Photometry done on 28 June 1971. U-B $=-0.65 \pm .19$ shows the object to be a quasar. Confirmed spectroscopically and redshift obtained by Baldwin et al. (1973).

TABLE 3

SIT PHOTOMETRY

\begin{tabular}{|c|c|c|c|c|c|c|c|}
\hline Cluster & Object & $z$ & $b^{\text {II }}$ & $\begin{array}{l}\text { Diameter } \\
\text { (arcsec) }\end{array}$ & $V$ & $B-V$ & $V-R$ \\
\hline A2100* . . & $\begin{array}{l}\text { N1 } \\
\text { N2 }\end{array}$ & 0.153 & +54 & $\begin{array}{l}\mathrm{SM}_{18.8}(24.3) \\
\end{array}$ & $\begin{array}{l}16.90 \\
17.31\end{array}$ & $\begin{array}{l}1.46 \\
1.42\end{array}$ & $\begin{array}{l}0.96 \\
1.01\end{array}$ \\
\hline $0822+67 \dagger($ IIIa-J) $\ldots \ldots \ldots$ & $\mathrm{G1}$ & $\begin{array}{l}0.384 \\
0.384\end{array}$ & +34 & SM (13.9) & $\begin{array}{l}20.0 \\
19.7\end{array}$ & $\ldots$ & $\begin{array}{l}0.64 \\
1.34\end{array}$ \\
\hline $1613+3104 \ddagger($ IIIa-J) $\ldots \ldots$ & $\begin{array}{l}F \\
G\end{array}$ & 0.415 & +46 & SM (13.5) & $\begin{array}{l}20.2 \\
19.6\end{array}$ & $\begin{array}{l}1.5: \\
1.47\end{array}$ & $\begin{array}{l}1.5 \\
1.45\end{array}$ \\
\hline 3C $299 \S \ldots \ldots$ & ${ }_{2}^{\text {ID }}$ & 0.367 & +67 & $\mathrm{SM}_{10}^{(14.2)}$ & $\begin{array}{l}19.43 \\
20.4\end{array}$ & $\begin{array}{l}0.93 \\
1.4\end{array}$ & $\begin{array}{l}1.36 \\
1.25\end{array}$ \\
\hline$\ldots \ldots \ldots \ldots$ & $\begin{array}{l}\text { ID } \\
\text { COMP }\end{array}$ & $\begin{array}{l}0.549 \\
0.532\end{array}$ & +41 & SM (12.3) & $\begin{array}{l}20.25 \\
20.40\end{array}$ & $\ldots$ & $\begin{array}{l}1.15 \\
1.66\end{array}$ \\
\hline $\begin{array}{l}3 \mathrm{3} 4438 \ldots \ldots \ldots \ldots \ldots \ldots \ldots \ldots \\
\text { 3C } 460 \# \ldots \ldots \ldots \ldots \ldots\end{array}$ & СӧмP & $\begin{array}{c}0.292 \\
0.268 \\
\ldots\end{array}$ & $\begin{array}{l}-13 \\
-35 \\
\ldots\end{array}$ & $\begin{array}{c}\text { SM (15.4) } \\
\text { SM (16.8) } \\
3.6\end{array}$ & $\begin{array}{l}19.18 \\
18.8 \\
22.1\end{array}$ & $\begin{array}{l}\cdots \\
\cdots \\
\cdots\end{array}$ & $\begin{array}{l}1.5: \\
1.3 \\
1.4:\end{array}$ \\
\hline
\end{tabular}
1956.

* Previous photoelectric $B$ and $V$ by Sandage $1972 b$. Red magnitude new here. Redshift by Humason, Mayall, and Sandage

$\dagger \mathrm{G} 1$ is the galaxy whose redshift was measured; A is the brightest cluster galaxy.

$\ddagger F$ is the galaxy whose redshift was measured (Paper I); $G$ is the brightest cluster galaxy.

$\S$ ID is the radio source identification and the brightest galaxy of the cluster (Kristian, Sandage, and Katem 1974), and may have some asymmetric structure; 2 is the second brightest galaxy. Redshift by Spinrad and Smith 1973.

$\|$ The two galaxies are discussed in the notes to Table 1 .

\# The companion is the faint object referred to by Kristian, Sandage, and Katem 1978. Cluster membership may be in doubt. 


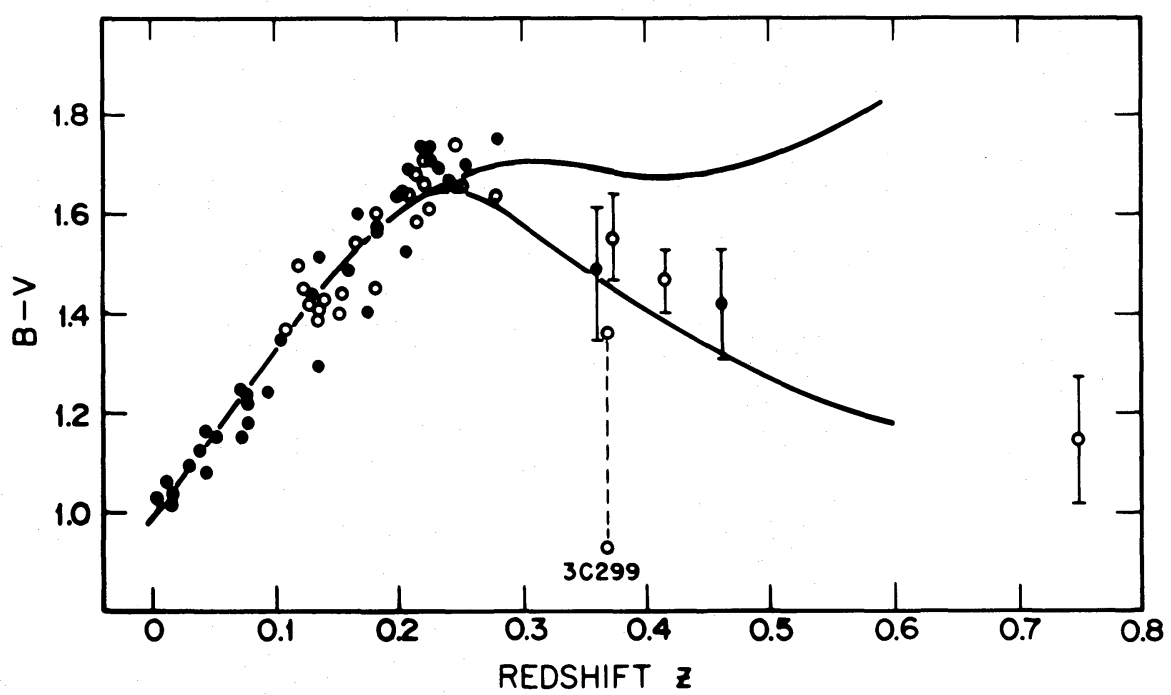

Fig. 1.-Measured $B-V$ colors versus redshift $z$ for the data sample defined by Tables 4 and 5 . New data are indicated by open circles (Table 4), older data (Table 5) by closed dots. The line to the left of $z \approx 0.25$ is that predicted from Whitford's (1975) standard-galaxy $K$ corrections. The two lines to the right are predicted values from the extremes of a sample of galaxies measured in the ultraviolet by Code and Welch (1977). The data suggest that the energy distributions of the galaxies in our sample are similar to that of NGC 4486, which defines the lower curve. Note that, at large redshift, the color turns over and becomes bluer: This supports a suggestion by $\mathbf{R}$. Kron that very distant clusters will be relatively blue.

The point labeled 3C 299 is the radio source, which is also the brightest cluster galaxy. The point above it, joined to it by a dashed line, is the second brightest galaxy of the cluster. The latter has colors which are similar to the rest of the sample, while the radio source is too blue by $\sim 0.5 \mathrm{mag}$ (see $\S \mathrm{III}$ ).

was proposed by Kron (1977) as a means of searching for extremely distant clusters. The present data are not very strong, but they support Kron's suggestion. For smaller redshifts, the Whitford $K$ corrections, as far as they are defined, appear to be a reasonable representation of the present data; this supports their use in $V$ and $R$ at least out to $z \approx 0.5$.
We note in passing the anomalously blue colors of 3C 299 in $B-V$ (Fig. 1) and of 3C 330 in $V-R$ (Fig. 2). In both cases, the second brightest cluster galaxy (joined to the radio source in Fig. 1 or 2 by a dashed line) has normal colors. The galaxy 3C 299 has a normal $V-R$ color; but this is accidental, since it has very bright [O III] emission (Spinrad and Smith

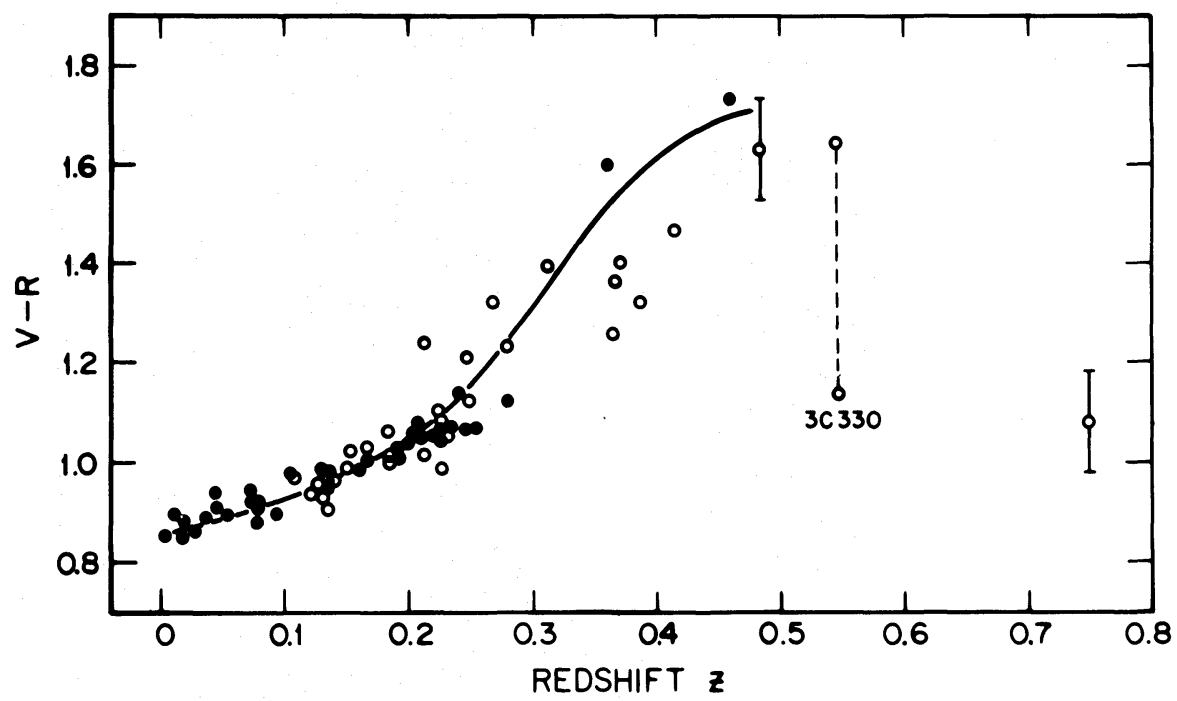

Fig. 2.-Measured $V-R$ colors versus redshift for the data sample defined in Tables 4 and 5 . Coding is the same as in Fig. 1 . The line is that predicted from Whitford's $K$ corrections. The prediction is a reasonable fit to the data, which suggests that the $K$ corrections for $V$ and $R$ are valid to $z \approx 0.5$. Note that the colors show the same turnover and increasing blueness with $z$ as do the $B-V$ data in Fig. 1 .

The point labeled 3C 330 is for the radio source, which is the brightest cluster galaxy in $V$. It appears too blue for its redshift. The point above it, joined to it by a dashed line, is for the second brightest galaxy (the brightest in $R$ ), which shows normal colors. 


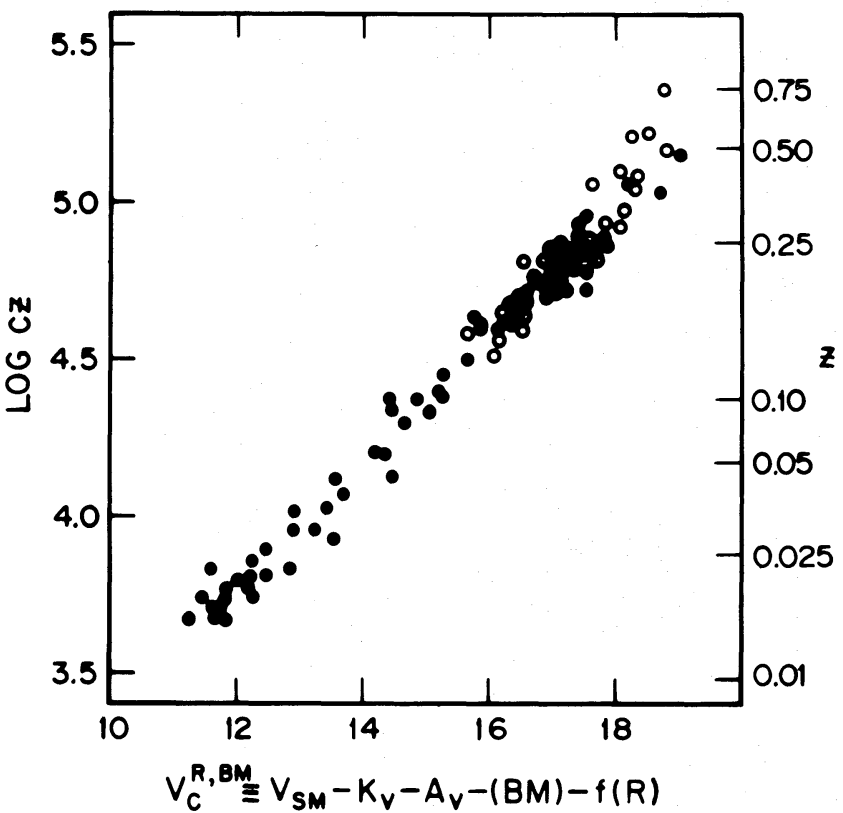

Fig. 3.-The Hubble diagram, in $V$, for the data sample of Tables 4 and 5. Open circles indicate data that are new in this paper; filled circles, earlier data. The following corrections have been applied to the magnitudes: an aperture correction to a standard metric diameter (SM), computed as if $q_{0}=+1$; Whitford's standard-galaxy $K$ correction $\left(K_{V}\right)$; a correction for galactic absorption $\left(A_{V}\right)$; and corrections for Bautz-Morgan contrast class (BM) and richness (R). The corrections are discussed in $\S$ IV. The data for $z<0.4$ show a small positive curvature (formal $q_{0}=+1.6$, when evolutionary effects are ignored), as discussed in $\S \mathrm{V}$.

1973) which makes the $R$ band too bright. Therefore, 3C 299 has a continuum which is too blue for a galaxy at its redshift over the entire spectral range (rest frame $\approx 3000-5500 \AA$ ) measured here. Both the $V$ and $R$ magnitudes of 3C 330 are also contaminated by a rich emission-line spectrum (Spinrad et al. 1976), but the continuum is probably still too blue in $V-R$; The galaxy was not measured in $B$.

\section{THE CORRECTED HUBBLE DIAGRAM}

Figures 3 and 4 summarize the present status of our program on the Hubble diagram for galaxy clusters.

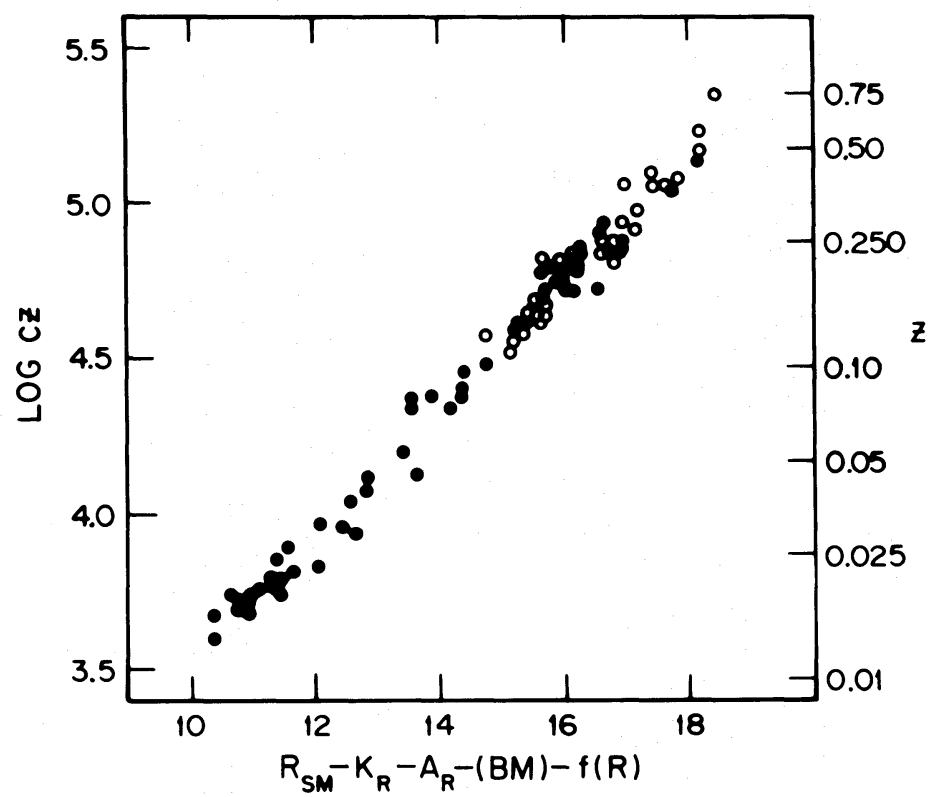

Fig. 4.- The same as Fig. 3, but for red magnitudes. Nearly identical formal $q_{0}$ values obtain from these $R$ data as from the $V$ data of Fig. 3. 
In addition to data for 32 clusters from the present paper, we include data for 16 clusters from Paper I and 55 clusters and groups from earlier work by

I Sandage, for a total sample of 103 clusters with redshifts ranging from 0.016 to 0.75 . Probably the most interesting aspect of the data is that they begin for the first time to suggest a departure from the $q_{0}=+1$ line.

In addition to the $K$ correction, discussed in $\S$ III, the data as plotted in Figures 3 and 4 have been subjected to several other standard corrections, which we will discuss briefly. The corrected data from Tables 2 and 3 of the present paper are given in Table 4, while the corrected data from the earlier work are given in Table 5.

\section{a) The Aperture Correction}

In Figures 3 and 4, we have used the aperture correction given earlier by Sandage (1972a, his Table 3), computed as if $q_{0}=+1$. But the aperture correction, which reduces measurements at different values of $z$ to the same intrinsic size at the galaxies, involves a dilemma in principle. To apply the correction properly, one must know the true value of $q_{0}$ beforehand, since the relation between linear size at a given $z$ and the apparent angular size at the Earth depends on it. Therefore, if the purpose of the measurements is to determine $q_{0}$, the possibility arises that one may be stalemated and unable to proceed. Whether this is a real problem in practice depends upon the sensitivity of the correction itself to the true value of $q_{0}$. If this sensitivity is not too great (i.e., if the aperture correction does not change much with a change in the true value of $q_{0}$ ), one can proceed by assuming a value of $q_{0}$ for purposes of the aperture correction and then computing a best-fit $q_{0}$ from the corrected data. If the assumed and computed values are not the same, one can then adjust the former and recompute the latter; this iterative procedure should converge to a self-consistent value of $q_{0}$ to the desired accuracy.

The question of the sensitivity of the results of the aperture correction to the value of $q_{0}$ was discussed briefly in Paper I. To explore the effect more fully and explicitly, we have independently applied two aperture corrections to our data: the corrections for assumed values of $q_{0}=0$ and $q_{0}=+1$. The differences can be seen in Tables 4 and 5 by comparing columns (5)-(8) with columns (9)-(12). The only difference between any corresponding pair of magnitudes in these tables-for example, between columns (5) and (9), or between columns (8) and (12)-is the difference of the assumed value of $q_{0}$ for the aperture correction. By comparing the two sets of data, we see that the corrected magnitudes are fainter for the $q_{0}=0$ case by $\sim 0.01 \mathrm{mag}$ for $z=0.04-0.06$, by $\sim 0.02 \mathrm{mag}$ for $z=0.07-0.12$, and by $0.02-0.06 \mathrm{mag}$ for $z=0.12$ 0.5 . The difference is not large, and its effects on the ultimate determination of $q_{0}$ are discussed further in $\S \mathrm{V}$.

TABLE 4

Reduction to Totally Corrected Magnitudes of the New Photometry in Tables 2 and 3

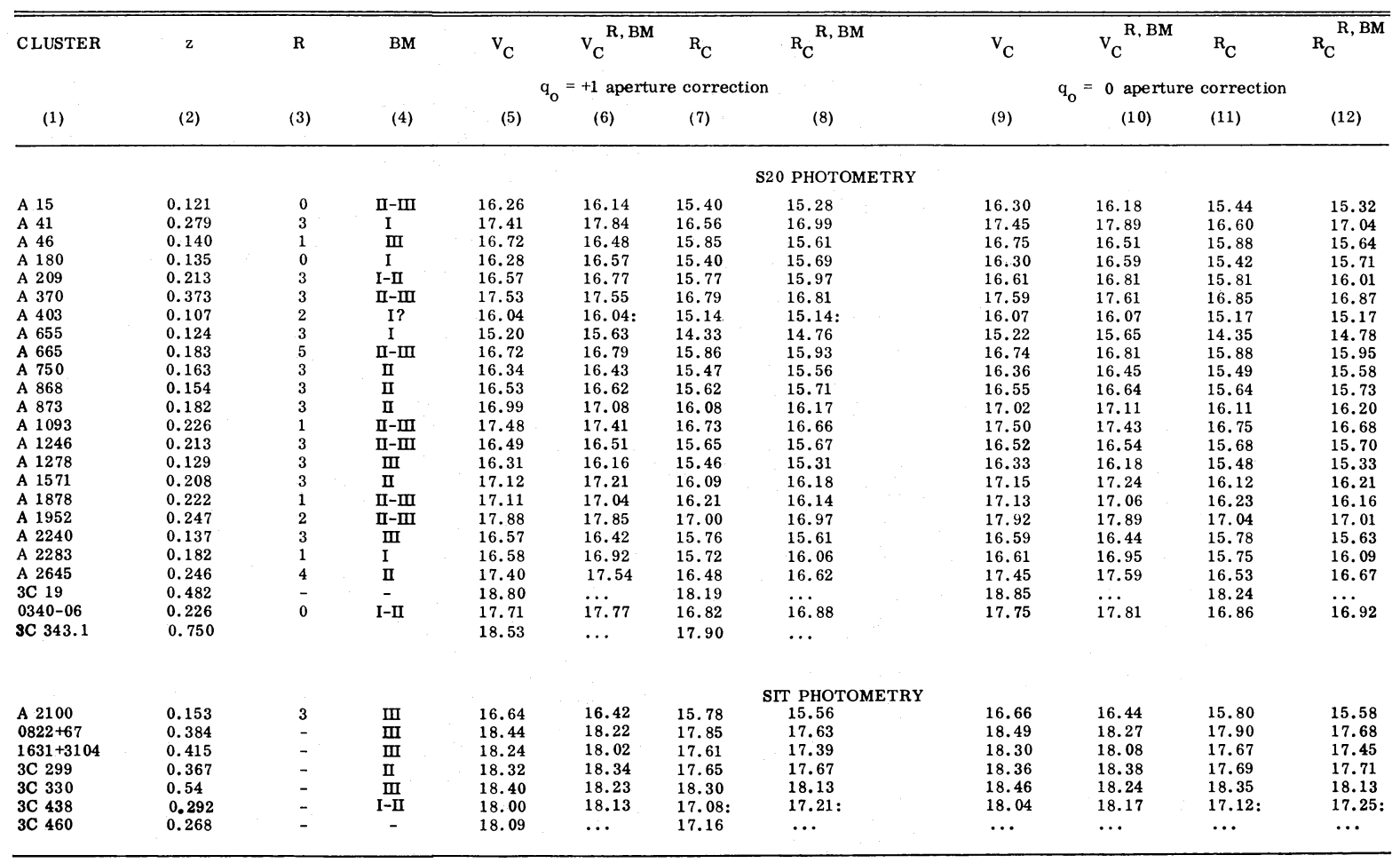


TABLE 5

Summary and New Reductions of Previously Studied Clusters with $v \geq 4000 \mathrm{~km} \mathrm{~s}^{-1}$

\begin{tabular}{|c|c|c|c|c|c|c|c|c|c|c|c|}
\hline CLUSTER & $\mathrm{z}$ & $\mathrm{R}$ & BM & $\mathrm{v}_{\mathrm{C}}$ & $v_{C}^{R, B M}$ & $\mathrm{R}_{\mathrm{C}}$ & $\mathrm{R}_{\mathrm{C}}^{\mathrm{R}, \mathrm{BM}}$ & $\mathrm{v}_{\mathrm{C}}$ & $\mathrm{v}_{\mathrm{C}}^{\mathrm{R}, \mathrm{BM}}$ & $\mathrm{R}_{\mathrm{C}}$ & $\mathrm{R}_{\mathrm{C}}^{\mathrm{R}, \mathrm{BM}}$ \\
\hline \multicolumn{8}{|c|}{$\mathrm{q}_{0}=+1$ aperture correction } & \multicolumn{4}{|c|}{$\mathrm{q}_{0}=0$ aperture correction } \\
\hline (1) & (2) & (3) & (4) & $(5)$ & (6) & (7) & $(8)$ & (9) & $(10)$ & (11) & $(12)$ \\
\hline \multicolumn{12}{|c|}{ THE SIXTEEN CLUSTERS FROM PAPER I (SKW) } \\
\hline A586 & 0.172 & 3 & I-II & 16.38 & 16.58 & 15.50 & 15.70 & 16.40 & 16.60 & 15.52 & 15.72 \\
\hline A593 & 0.226 & 3 & II-III & 17.46 & 17.49 & 16.70 & 16.73 & 17.50 & 17.53 & 16.74 & 16.77 \\
\hline A639 & 0.291 ? & 3 & II-III & 17.48 & 17.50 & $\ldots$ & $\ldots$ & 17.52 & 17.54 & $\ldots$ & $\ldots$ \\
\hline A777 & 0.224 & 4 & II-III & 17.49 & 17.56 & 16.84 & 16.91 & 17.52 & 17.59 & 16.87 & 16.94 \\
\hline A795 & 0.136 & 3 & II-III & 16.29 & 16.31 & 15.42 & 15.44 & 16.32 & 16.34 & 15.45 & 15.47 \\
\hline A963 & 0.206 & 3 & II & 16.97 & 17.06 & 15.76 & 15.85 & 16.99 & 17.08 & 15.78 & 15.87 \\
\hline A1146 & 0.137 & 4 & $\mathrm{I}-\mathrm{II}$ & 15.98 & 16.23 & 15.02 & 15.27 & 16.00 & 16.25 & 15.04 & 15.29 \\
\hline A1514 & 0.200 & 3 & II-III & 17.05 & 17.07 & 16.18 & 16.20 & 17.09 & 17.11 & 16.22 & 16.24 \\
\hline A1550 & 0.254 & 3 & II-III & 17.34 & 17.36 & 16.57 & 16.59 & 17.38 & 17.40 & 16.61 & 16.63 \\
\hline A1758 & 0.281 & 3 & III & 17.51 & 17.36 & 16.78 & 16.63 & 17.55 & 17.40 & 16.82 & 16.67 \\
\hline A1759 & 0.168 & 3 & III & 17.17 & 17.02 & 16.19 & 16.04 & 17.20 & 17.05 & 16.22 & 16.07 \\
\hline A1957 & 0.241 & 3 & $(\mathrm{I}-\mathrm{II})$ & 17.63 & 17.83 & 16.75 & 16.95 & 17.67 & 17.87 & 16.79 & 16.99 \\
\hline A1961 & 0.236 & 3 & II-III & 16.91 & 16.93 & 16.27 & 16.29 & 16.95 & 16.97 & 16.31 & 16.33 \\
\hline A2111 & 0.228 & 3 & III & 17.37 & 17.22 & 16.43 & 16.28 & 17.41 & 17.26 & 16.47 & 16.32 \\
\hline A2125 & 0.246 & 4 & II-III & 17.07 & 17.14 & 16.88 & 16.95 & 17.09 & 17.16 & 16.90 & 16.97 \\
\hline $0939+09$ & 0.206 & (3) & II-III & 17.15 & 17.17 & 16.27 & 16.29 & 17.18 & 17.20 & 16.30 & 16.32 \\
\hline
\end{tabular}

FROM TABLE 4 of PAPER VI (S) AND TABLE 6 OF PAPER VII (SH)

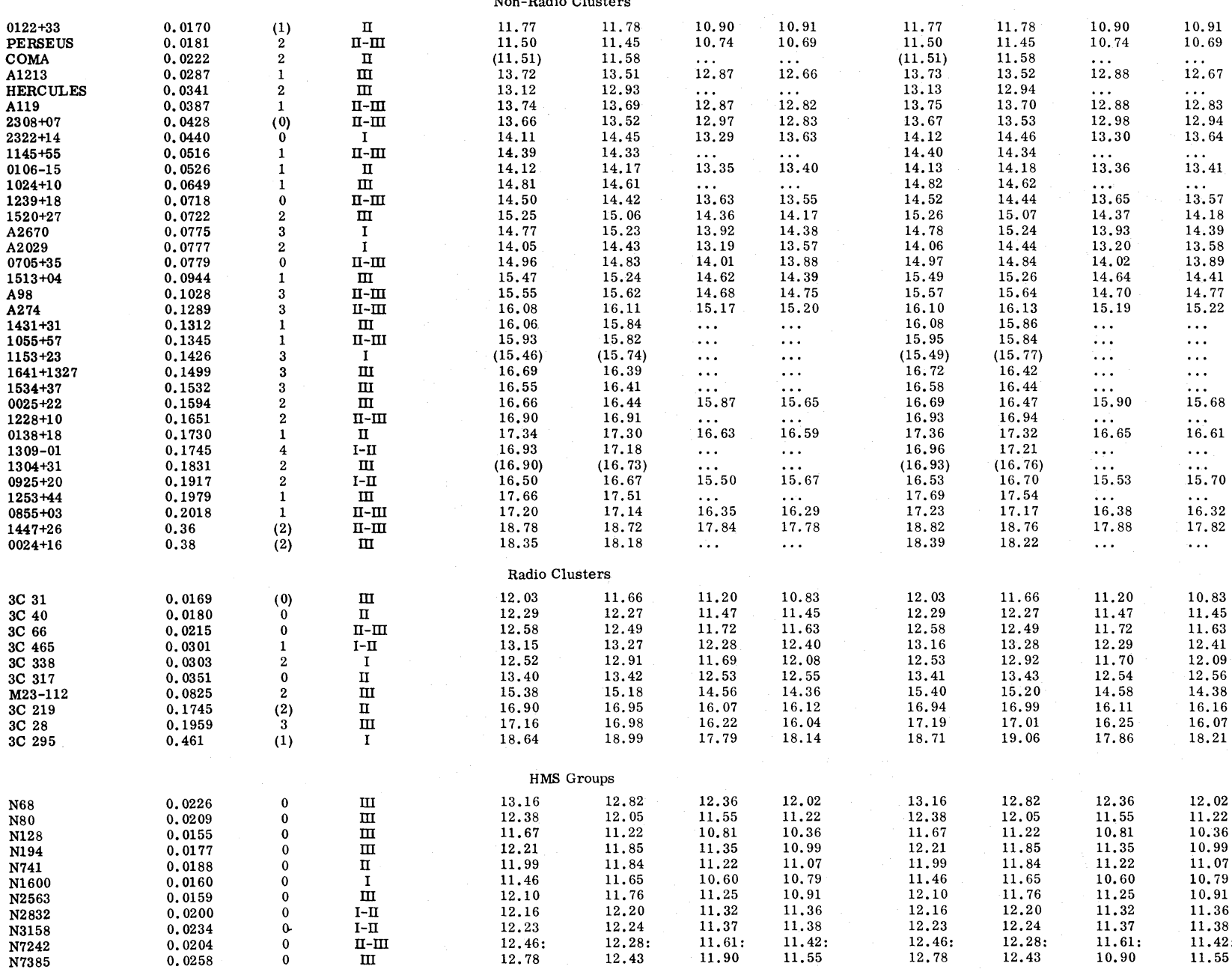


b) Corrections for Richness, Bautz-Morgan Class, and Galactic Absorption

We have corrected the final magnitudes in Tables 4 and 5 and Figures 1 and 2 for Abell richness class according to the recipe of Table 3 of Paper I, for Bautz-Morgan contrast class following the discussion of Sandage and Hardy (1973), and for galactic absorption as defined by Sandage (1973a, his eqs. [4] and [5]).

The addition of the new data here does not significantly alter the earlier discussion of either the richness or Bautz-Morgan correction. The main impact of the new data for the richness correction is in the redshift range $0.10<z<0.27$, with the addition of 10 new clusters of richness 3 and the inclusion for the first time of clusters of richness 0 (three clusters) and richness 5 (the richest Abell cluster, A665). The richness effect is in any case small, and the new data appear to be adequately represented by the discussion of Paper I. We have not tried to estimate richness. classes for the most distant clusters because of the difficulty of seeing deep enough into the cluster luminosity function for these very faint clusters. We do not believe that one will necessarily tend to selectively discover richer clusters at very large redshifts. It seems more likely that discovery will most strongly depend on the Bautz-Morgan contrast class: There will be a tendency to notice clusters where the bright end of the luminosity function is well populated (larger BM classes). This tendency can perhaps already be seen in Table 4.

The correlation between residuals from the mean Hubble line and Bautz-Morgan class continues to hold for our enlarged data sample. For the combined data of Sandage and Hardy (1973), Paper I, and the present paper, we obtain the following mean residuals: $\mathrm{BM} \mathrm{I}=-0.37 \pm 0.09 \mathrm{mag}$ (19 clusters); BM I-II $=-0.13 \pm 0.08 \mathrm{mag}$ (14); BM II $=-0.04$ $\pm 0.07 \mathrm{mag}(21) ; \mathrm{BM}$ II-III $=+0.04 \pm 0.06 \mathrm{mag}$ (35); and $\mathrm{BM}$ III $=+0.17 \pm 0.04 \mathrm{mag}(5 \overline{2})$.

\section{c) Radio Sources in the Sample}

The data include redshifts and magnitudes for 13 bright cluster galaxies that are also radio sources and four radio galaxies that may or may not be in clusters (3C 19, 3C 123, 3C 343.1, and 3C 460). The use of these objects in a discussion of the Hubble diagram may be questioned because of (1) evidence for a relation between optical and radio absolute luminosities of radio galaxies (e.g., Colla et al. 1975; Jaffe and Perola 1976), combined with a strong bias toward detection of radio-bright objects at large redshifts, and (2) the possibility that some radio sources may have significant nonthermal radiation or other anomalies in the visible region of the spectrum.

The present data suggest that item (1) is not important for cluster galaxies but that item (2) may be, especially at extreme redshifts. For redshifts less than 0.5 , there is no discernible difference between the systematics of the radio and nonradio clusters in our sample as a function of $z$. The reason that the relation between radio and optical luminosities is not important for clusters appears to be its form: As one goes to lower radio luminosities, the distribution of optical luminosities becomes increasingly more skewed by extending further to low optical luminosities, but the magnitude and shape of the bright end remain the same. This effect can be seen explicitly in Figure 6 of Colla et al. (1975) and also appears in the 3CR sample of Sandage (1972c, his Table 2). It seems that, by considering only radio sources that are also the brightest cluster galaxies, we restrict ourselves to the (constant) high-luminosity end of the optical distribution. Stated differently and more simply, it appears empirically that, to within the uncertainties of the present data, the brightness of the brightest cluster galaxy does not depend on whether that galaxy is also a bright radio source. For noncluster galaxies, however, there may well be an effect. Our data are not compatible with the prediction by Gunn (1977) of a large effect. The reason seems to be that Gunn represented the Colla et al. data by a linear relation between radio and optical magnitudes, which is probably not an adequate representation of the relation discussed above.

\section{THE APPARENT VALUE OF $q_{0}$ WITH NO EVOLUTIONARY CORRECTION: EVIDENCE FOR A POSITIVE CURVATURE OF THE HUBBLE LINE}

It is clear from a cursory inspection of Figures 3 and 4 that the Hubble line continues to be approximately straight, and the dispersion in magnitude reasonably uniform, to the limit of the available data. But a closer examination of the data shows, for the first time, a persistent positive curvature of the line.

Table 6 gives the results of a formal least-squares fit of several sets of data to a Friedmann model of the universe, with all galaxy evolution explicitly ignored. To avoid the possibility of distorting the results by the few largest redshift objects, for which the corrections are greatest and most uncertain, and for which selection effects are probably operative (especially a tendency to selectively observe objects on the brighter side of the luminosity function at extreme redshifts), we have restricted the discussion to clusters with redshift $z<0.4$. In recent years, this redshift range has become rather easy to work in: The data are secure, and a reasonable data sample is available which is not limited in apparent luminosity. Except for the $3 \mathrm{C}$ clusters, the sample is a random selection from the rich Abell and deeper Schmidt survey clusters.

The first data sample in Table 6 consists of totally corrected magnitudes for all galaxies in Tables 4 and 5 , columns (6) and (8), with $z<0.4$ and the $q_{0}=+1$ aperture correction. For the $V$ and $R$ magnitudes separately, we have computed the least-squares values of the deceleration parameter $q_{0}$ and the mean absolute magnitude, together with their standard deviations. Also included in Table 6 are the rms deviation of the magnitudes about the best-fit line $(\sigma)$ and the number of clusters in the sample $(n)$.

Sample 2 consists of sample 1 with the addition of 43 low-redshift clusters from Sandage and Hardy (1973), which should have the effect of more securely 
TABLE 6

Least-Squares Values of $q_{0}$ For Several Data Samples: $z<0.4$

\begin{tabular}{|c|c|c|c|c|c|c|c|c|c|c|}
\hline \multirow[b]{2}{*}{ Data Sample } & \multicolumn{4}{|c|}{$\mathrm{V}$ magnitudes } & \multirow[b]{2}{*}{$\mathrm{n}$} & \multicolumn{5}{|c|}{ R magnitudes } \\
\hline & $q$ & $\mathrm{o}_{0}$ & $\sigma$ & $\begin{array}{l}\left\langle\mathrm{V}_{\mathrm{abs}}\right\rangle \\
\mathrm{H}_{\mathrm{O}}=50\end{array}$ & & & $q_{0}$ & $\tau$ & $\begin{array}{l}\left\langle\mathrm{R}_{\mathrm{abs}}\right\rangle \\
\mathrm{H}_{\mathrm{o}}=50\end{array}$ & $\mathrm{n}$ \\
\hline 1 & 1.63 & $\begin{array}{l}+.48 \\
-.42\end{array}$ & $0 . \mathrm{m}_{30}$ & $-23 m^{m_{2}} 26 \pm .05$ & 96 & 1.61 & $\begin{array}{l}+.50 \\
-.42\end{array}$ & $0 . \mathrm{m}_{28}$ & $-24 . .^{\mathrm{m}} 09 \pm .05$ & 83 \\
\hline 2 & 1.57 & $\begin{array}{l}+.35 \\
-.32\end{array}$ & 0.28 & $-23.28 \pm .03$ & 139 & 1.66 & $\begin{array}{l}+.39 \\
-.35\end{array}$ & 0.27 & $-24.09 \pm .03$ & 118 \\
\hline 3 & 1.53 & $\begin{array}{l}+.53 \\
-.47\end{array}$ & 0.31 & $-23.29 \pm .06$ & 85 & 1.54 & $\begin{array}{l}+.57 \\
-.50\end{array}$ & 0.30 & $-24.11 \pm .06$ & 70 \\
\hline 4 & 1.44 & $\begin{array}{l}+.45 \\
-.40\end{array}$ & 0.30 & $-23.26 \pm .05$ & 96 & 1.43 & $\begin{array}{l}+.47 \\
-.42\end{array}$ & 0.28 & $-24.09 \pm .05$ & 82 \\
\hline 5 & 2.21 & $\begin{array}{l}+.66 \\
-.56\end{array}$ & 0.35 & $-23.18 \pm .06$ & 97 & 2.34 & $\begin{array}{l}+.71 \\
-.63\end{array}$ & 0.34 & $-24.00 \pm .07$ & 82 \\
\hline
\end{tabular}

\section{Definition of Data Samples in Table 6}

1. Tables 4 and 5 of this paper, totally corrected magnitudes, $q_{0}=+1$ aperture correction, $z<0.4$.

2. Sample 1 plus the remainder of Sandage and Hardy (1973) Table 6 not already included in Sample 1 (mostly small redshift clusters).

3. Sample 1 with all radio sources omitted.

4. The galaxies of sample 1 with the $q_{0}=0$ aperture correction.

5. Sample 1, but magnitudes not cgrrected for richness or Bautz-Morgan class.

anchoring the bright end. The best-fit values for $q_{0}$ and $M_{\text {abs }}$ are essentially identical with those of sample 1 , and the standard deviations are slightly decreased.

The values of $q_{0}$ found here are for the first time notably different from +1 . The value $q_{0}=+1$ is $1.5-2 \mathrm{~s}$.d. from the best fit; the value $q_{0}=+0.5$ (the just-closed Friedmann model, if no evolution is assumed) is about 3 s.d. from the best fit. It should be noted that the dispersion in magnitude, which is real, continues to be remarkably small $(0.3 \mathrm{mag})$ and is uniform in $z$ (see Figs. 3 and 4). Also, Table 6 shows that the separate results for $V$ and $R$ magnitudes are identical, which implies both that the Whitford $K$ corrections are valid to $z=0.4$ and that there has been no significant color evolution over this range.

Samples 3-5 of Table 6 show the results of various corrections on the value of $q_{0}$. Sample 3 is the same as sample 1 with all radio sources omitted. The results are negligibly different from those of sample 1 . This supports the statement above $(\S \mathrm{IV} c)$ that the brightness of the brightest cluster galaxy is the same whether or not it is a strong radio source.

Sample 4 shows the effect of the dependence of the aperture correction on the value of $q_{0}$ itself (see $\S \mathrm{IV} a$ above). This sample consists of the same galaxies as does sample 1 with the $q_{0}=0$ aperture corrections (cols. [10] and [12] of Tables 4 and 5). The effect is to reduce the computed values of $q_{0}$ by 0.2 . It is clear from these numbers that the dependence of the aperture correction on $q_{0}$ does not preclude finding $q_{0}$ from such data; a few iterations will quickly converge to a self-consistent value of $q_{0}$.

The basic reason that the aperture correction is not a strong function of $q_{0}$ is that our standard aperture size is so large that the integrated intensity curve $I(r)$ is rather flat, as discussed in Paper I.

Finally, sample 5 illustrates the effect of the richness and Bautz-Morgan corrections. Sample 5 is the same as sample 1 but without these two corrections (cols. [5] and [7] of Tables 4 and 5). As can be seen from Table 6 , the effect of the richness plus BM corrections is to decrease the best-fit value of $q_{0}$ (in the absence of these corrections, the computed $q_{0}$ is larger) and at the same time to reduce the dispersion of the magnitude residuals significantly.

In an earlier discussion of an inhomogeneous set of data, consisting of broad-band measurements by Sandage $(1972 b, 1973 a)$ and Peterson (1970) for $z<0.1$ and their own scanner data for $z=0.2-0.4$, Gunn and Oke (1975) concluded that the Hubble line showed a negative curvature $\left(q_{0}\right.$ between +0.33 and -1.27). Largely on the basis of this result and with an assumed evolutionary model, Gunn and Tinsley (1975) then took the argument a step farther and suggested that the universe might be accelerating, driven by a nonzero cosmological constant.

Our present data, which are basically an extension of the earlier Sandage broad-band data (and include the Peterson data in sample 2), do not confirm the Gunn and Oke results. This situation is epitomized by the case of 3C 295, the most distant Gunn and Oke galaxy, which they found to be "an extreme example... it lies well above the mean absolute magnitude in any fit." On the basis of the present sample, however, which goes beyond 3C 295, this galaxy does not appear to be abnormally bright; it 
lies about $0.2 \mathrm{mag}$ to the right of the mean Hubble line, which is within the magnitude dispersion, and on the faint side. This result, moreover, is independent of the corrections applied to the data, since 3C 295 is slightly fainter than other cluster galaxies in the same general redshift range, both radio sources and radio-quiet galaxies.

\section{DISCUSSION}

After nearly 50 years, the original Hubble diagram has been extended by a factor greater than 200 in redshift, and the data have begun to show a significant departure from the linearity expected for all models in the limit of small redshift. The usefulness of this classical approach to testing world models now depends on an accurate knowledge of changes with time of the luminosity of elliptical galaxies. How accurate this knowledge must be depends upon the true value of $q_{0}$, since the most important question at present is a qualitative one: Is the universe open or closed? The nearer $q_{0}$ is to the critical value of $\frac{1}{2}$, the more accurately it must be determined.

If it were known with certainty, for example, that there has been no significant luminosity change during the last one-fourth of the Hubble time $\left(4 \times 10^{9}\right.$ years, if $H_{0}=55 \mathrm{~km} \mathrm{~s}^{-1} \mathrm{Mpc}^{-1}$ ), then the present data are nearly good enough to show that the universe is closed. The values of $q_{0}$ for sample 1 or 2 of Table 6 would in this case have to be increased slightly to $q_{0} \approx 1.7 \pm 0.4$, and the just-closed case would be 3 s.d. from the best value. The present age of the universe would then be $12 \times 10^{9}$ years (with $H_{0}=$ $55 \mathrm{~km} \mathrm{~s}^{-1} \mathrm{Mpc}^{-1}$ ), and it would be expected to have collapsed again about $60 \times 10^{9}$ years hence.

Another possibility is that the value of $q_{0}$ may be definitively found in some other way, in which case the Hubble diagram can then be used to determine the total rate of change of galaxy luminosity and thus to set an important constraint upon possible models of galaxy evolution. If, for example, it were known with certainty that $q_{0} \approx 0$, then sample 4 of Table 6 is the correct one, and it requires a dimming of about 0.5 mag during the last $5 \times 10^{9}$ years, with no major color changes (say, $\Delta[B-V] \leqslant 0.1 \mathrm{mag}$ ). Simple evolving galaxy models (Sandage 1961; Tinsley and Gunn 1976) can be made consistent with such changes, but the question of possible dynamical evolution (Ostriker and Tremaine 1975) is still open.

Several lines of evidence suggest that the value of $q_{0}$ may in fact be small (see $\S$ VI of Sandage 1975 and the references therein). Taken piecemeal, none of the evidence is compelling at present; but the fact that several independent kinds of data point in the same direction perhaps lends more weight to the total.

In any case, it appears possible at present to construct a self-consistent picture of an open and nearly empty universe which accommodates the little that is known without much strain; but the basis of such a picture is not very secure, and, in the face of one really solid piece of evidence that $q_{0}=+1$, say, it could be given up without major dislocations.

We thank Dr. A. D. Code for kindly sending us a preprint of the Code and Welch paper, and Drs. L. Woltjer and F. Pacini for their hospitality during a visit by $\mathrm{J}$. K. to ESO, where part of this paper was written.

This work was supported in part by a grant from the National Science Foundation (MPS 75-16327) to the Carnegie Institution of Washington.

\section{REFERENCES}

Baldwin, J. A., Burbidge, E. M., Hazard, C., Murdoch, H. S., Robinson, L. B., and Wampler, G. J. 1973, Ap. J., 185, 739.

Code, A. D., and Welch, G. A. 1977, preprint.

Colla, G., Fanti, C., Fanti, R., Gioia, I., Lari, C., Lequeux, J., Lucas, R., and Ulrich, M.-H. 1975, Astr. Ap., 38, 209.

Gunn, J. E. 1977, in IAU Colloquium No. 37, Redshifts and the Expansion of the Universe, ed. C. Balkowski and B. E. Westerlund (Paris: CNRS), p. 183.

Gunn, J. E., and Oke, J. B. 1975, Ap. J., 195, 255

Gunn, J. E., and Tinsley, B. M. 1975, Nature, 257, 454.

Humason, M. L., Mayall, N. V., and Sandage, A. R. 1956, A.J., 61, 97.

Jaffe, W. J., and Perola, G. C. 1976, Astr. Ap., 46, 275.

Kristian, J., Sandage, A., and Katem, B. N. 1974, Ap. J., 191, 43.

1978, Ap. J., 219, 803.

Kron, R. 1977 , personal communication.

Longair, M. S., and Gunn, J. E. 1975, M.N.R.A.S., 170, 121.

Ostriker, J. P., and Tremaine, S. D. 1975, Ap. J. (Letters), 202, L113.

Peterson, B. A. 1970, A.J., 75, 695.

Sandage, A. 1961, Ap. J., 134, 916.

-. 1972a, Ap.J., 173, 485 .

Sandage, A. 1972c, Ap. J., 178, 25.

-. 1973a, Ap. J., 183, 711. 1973b, Ap. J., 183, 731 .

-. 1975, Ap. J., 202, 563.

Sandage, A., and Hardy, E. 1973, Ap. J., 183, 743.

Sandage, A., Kristian, J., and Westphal, J. A. 1976, Ap. J., 205, 688 (Paper I).

Spinrad, H. 1975, Ap. J. (Letters), 199, L3.

Spinrad, H., Liebert, J., Smith, H. E., and Hunstead, R. 1976, Ap. J. (Letters), 206, L79.

Spinrad, H., and Smith, H. E. 1973, Ap. J. (Letters), 179, L71.

Spinrad, H., Westphal, J., Kristian, J., and Sandage, A. 1977, Ap. J. (Letters), 216, L87.

Tinsley, B. M., and Gunn, J. E. 1976, Ap. J., 203, 52.

Westphal, J. A., and Kristian, J. 1976, in IAU Colloquium No. 40, Astr. Applications Image Detectors with Linear Response, ed. M. Duchesne and G. Lelièvre (Meudon: Obs. de Paris), p. 19-1.

Westphal, J. A., Kristian, J., and Sandage, A. 1975, Ap. J. (Letters), 197, L95.

Whitford, A. E. 1975, in Galaxies and the Universe, ed. A. and M. Sandage and J. Kristian (Chicago: University of Chicago Press), p. 159.

Wyndham, J. D. 1966, Ap. J., 144, 459.

Jerome Kristian and Allan Sandage: Hale Observatories, 813 Santa Barbara Street, Pasadena, CA 91101

JAmes A. Westphal: California Institute of Technology, 1201 East California Boulevard, Pasadena, CA 91125 